\title{
Desarrollo de un Modelo Matemático preliminar para la detección de cáncer de próstata en los pacientes con biopsias prostáticas de repetición
}

\author{
Ramírez Backhaus M, Trassierra Villa M, Bahilo Mateu P, Pontones Moreno JL, \\ Santamaría Navarro C, Ruiz-Cerdá JL, Vera Donoso CD, Jiménez Cruz JF.
}

\author{
Servicio de Urología. Hospital Universitario La Fe. Valencia.
}

Actas Urol Esp. 2008;32(3):281-287

\begin{abstract}
RESUMEN
DESARROLLO DE UN MODELO MATEMÁTICO PRELIMINAR PARA LA DETECCIÓN DE CÁNCER DE PRÓSTATA EN LOS PACIENTES CON BIOPSIAS PROSTÁTICAS DE REPETICIÓN

Objetivo: Analizamos las variables clínicas e histopatológicas de los pacientes hombres a biopsiar por sospecha de cáncer de próstata y cuáles de ellos tienen carácter pronóstico para los pacientes con biopsia previa sin hallazgos de neoplasia. Pretendemos desarrollar un nomograma que nos ayude en la decisión de indicar la repetición de la prueba.

Material y método: Incluimos 179 pacientes con, al menos una biopsia previa sin hallazgos neoplásicos. Registramos, antes de cada biopsia, edad del paciente, antígeno próstatico específico (PSA) total, PSA libre/total, densidad del PSA, velocidad del PSA, tacto rectal, volumen ecográfico, aparición de área sospechosa en la escala de grises durante la ecografía transrectal, número de cilindros biopsiados, lesiones histológicas premalignas en alguna de las biopsias previas, así como el tiempo transcurrido entre las biopsias. Mediante un modelo de regresión logística determinamos la asociación de cada variable con la presencia de cáncer (biopsia positiva). Construimos un nomograma con las variables estadísticamente más relevantes y averiguamos la capacidad de discriminación del modelo mediante el índice de concordancia.

Resultados: Nuestras biopsias de repetición consiguen una tasa de detección de cáncer del 46\%. En el estudio univariante la edad, el tacto rectal, el volumen prostático, la densidad del PSA, las zonas sospechosas en la escala de grises, y las lesiones histológicas premalignas se asocian a biopsia de repetición positiva para cáncer (p <0.05). En el análisis multivariante, la edad, el tacto rectal, el volumen prostático y los antecedentes histológicos de una lesión preneoplásica se asociaron a biopsia positiva. Construimos un nomograma con un índice de concordancia de 0,80 .

Conclusión: A expensas de una validación prospectiva externa de nuestro modelo, el nomograma desarrollado podría ser de ayuda en la difícil de tarea de indicar una biopsia de repetición.
\end{abstract}

Palabras clave: Cáncer de próstata. Biopsia prostática. Nomograma.

\section{ABSTRACT}

A PRELIMINAR MATHEMATICAL MODEL FOR PATIENTS WITH A PREVIOUS NEGATIVE PROSTATE BIOPSY

Introduction and objectives: It is usual to identify patients with a negative prostate biopsy who are still at risk of prostate cancer. We try to analyse if the classical variables used in the prostate cancer screening are useful for those patients with a previous negative prostate biopsy, and if there is a possibility for making a nomogram witch would help us in the decision to repeat the biopsy.

Material and methods: We studied 179 patients with at least 1 initial negative biopsy. At each biopsy session we recorded: Patient age, serum prostate specific antigen (PSA), free PSA/total PSA, PSA slope, digital rectal examination, prostate volume, PSA density, cancer suspicion in previous transrectal ultrasounds findings, number of negative cores previously obtained, history of precarcinomatous lesions and time between biopsies. Through Logistic regression analysis we determined the association of each variable a positive biopsy. A nomogram was constructed using all variables and discrimination was calculated as the concordance index.

Results: Overall $46 \%$ of patients had cancer at the repeated biopsy session. In the univariate analysis: Age, digital rectal examination, prostate volume, PSA density, cancer suspicion in ultrasounds findings, and precarcinomatous lesions were associated with repeat positive biopsy for cancer (all $\mathrm{p}<0.05$ ). In the multivariate study, age, digital rectal examination, prostate volume and history of precarcinomatous lesions were associated with repeat positive biopsy. A nomogram was constructed that had a concordance index of 0.80 .

Keywords: Prostate cancer. Prostate Biopsy. Nomogram. 
$\mathrm{D}$ esde el descubrimiento y la utilización del antígeno prostático específico (PSA) como método de cribado del cáncer de próstata el número pacientes que precisan una biopsia de próstata ha aumentado considerablemente ${ }^{1}$. Pese a que está por demostrar el beneficio del cribado masivo de cáncer de próstata mediante PSA, lo que si parece evidente es que ha disminuido la incidencia de cáncer de próstata avanzado ${ }^{2}$. Dado que el PSA no es un marcador cáncer específico, en muchas ocasiones un aumento del los valores del PSA no traduce la presencia de cáncer, sino de hiperplasia prostática o procesos inflamatorios. Actualmente, se están investigando nuevas moléculas y estrategias para aumentar la especificidad del PSA, por ejemplo: cociente PSAlibre/ PSAtotal, PSA densidad, PSA edad ${ }^{3}$, y los anticuerpos anti $\mathrm{p} 53^{4}$, y también la utilidad de redes neuronales o nomogramas que combinan diferentes variables y que seleccionan a los pacientes con mayor probabilidad de padecer un cáncer de próstata ${ }^{5,6}$. No obstante, pese a todos estos esfuerzos, se siguen practicando muchas biopsias de próstata realmente innecesarias y la sensibilidad de la biopsia de próstata, independientemente de la estrategia practicada, está lejos de alcanzar el 100\%.

En la práctica clínica diaria es frecuente que nos encontremos con pacientes en los que persiste la sospecha de cáncer de próstata aunque se haya realizado una biopsia prostática cuyo resultado fuera negativo. De hecho en la literatura existen series con altas tasas de detección de cáncer en biopsias de repetición.

Nos planteamos en ese momento, con las variables clínico-patológicas que manejamos habitualmente, si esperar o insistir con una prueba, sin duda molesta, y en ocasiones con complicaciones asociadas; sobre todo con el aumento en el número de cilindros, que se obtienen al realizan biopsias de repetición.

Dado que en nuestro centro no están protocolizadas todas las indicaciones para una segunda biopsia, nos propusimos analizar, en los pacientes en los que se produce una elevación del PSA con biopsias previas negativas, el valor pronóstico de esas variables, así como identificar las que poseen la suficiente capacidad predictiva para establecer un modelo matemático que permita crear un nomograma útil en la indicación de re-biopsiar a los pacientes con biopsias previas negativas para cáncer.

\section{MATERIAL Y MÉTODO}

Desde el año 1990 a diciembre del año 2005 se han realizado en nuestro centro 342 biopsias de repetición. Se excluyeron aquellos pacientes en los que alguna biopsia se ha realizado fuera de nuestro centro, o pacientes con historiales clínicos incompletos.

Se estudiaron como posibles factores predictivos del cáncer de próstata: Edad, PSA total, PSA libre/ total, densidad del PSA, velocidad del PSA, tacto rectal, volumen ecográfico, aparición de área sospechosa en la escala de grises durante la ecografía transrectal, número de cilindros biopsiados, lesiones histológicas premalignas en alguna de las biopsias previas, así como el tiempo transcurrido entre las biopsias.

En primer lugar, se llevó a cabo un análisis descriptivo de los datos donde los resultados se expresan como media ( \pm desviación estándar) para variables cuantitativas y con proporciones para las variables cualitativas.

En segundo lugar y con el objetivo de determinar los posibles factores predictores de biopsia de repetición positiva para cáncer, se analiza la posible relación de cada una de las variables independientes consideradas con el resultado de la biopsia. Para ello se aplicó la regresión logística univariante con cada una de las variables, determinando la odds ratio con los intervalos de confianza al 95\%. Las variables que mostraron una significación estadística en cada uno de los modelos univariantes se introdujeron conjuntamente en un modelo de regresión logística multivariante.

Por último se elaboró un nomograma como representación gráfica del modelo de regresión logístico multivariante obtenido.

En todos los cálculos se aceptó como nivel de significación un valor de $p$ inferior a 0,05 y han sido realizados con la librería Design del programa SPlus (PC version Professional; Insightful Corp, Redmond, WA) y el programa SPSS 12.0.

Para el análisis estadístico multivariante englobamos las lesiones anatomopatológica tipo proliferación microacinar atípica y PIN como lesiones premalignas.

Para cuantificar la capacidad predictiva del nomograma se ha empleado el índice de concordancia de Harrel. Este índice oscila de 0,5 a 1. El valor 1 indicaría perfectas predicciones, mientras que el 
valor 0,5 indica predicciones aleatorias. Este índice muestra la capacidad que posee el nomograma para discriminar los riesgos de los pacientes, de forman que aquellos que presenten un peor pronóstico sean los más probables de experimentar una biopsia positiva.

La biopsia de próstata se realizaba transrectal con un ecógrafo siemens sonoline Antares ${ }^{\circledR}$ y una aguja de punción Boston ${ }^{\circledR}$ de 18 g. Según nuestro protocolo habitual, a los pacientes se les aplica enemas de limpieza la noche anterior y la mañana de la prueba, 30 minutos antes de la intervención se les administra una dosis de ciprofloxacino $400 \mathrm{mg}$ vía endovenosa y tobramicina $100 \mathrm{mg}$ como profilaxis antibiótica. Hasta septiembre del 2005 se biopsiaba según la técnica en sextante clásica, a la que se añadía un cilindro en el caso de existir alguna zona sospechosa en la escala de grises en la ecografía transrectal. A partir de dicha fecha se procedió a protocolizar las biopsias de repetición con toma de 14 cilindros que se distribuían de la siguiente manera: Seis cilindros según la técnica sextante clásica, dos cilindros de cada una de las zonas más laterales de la próstata, cuernos laterales, y dos cilindros de cada una de las zonas transicionales (referenciar mi artículo).

\section{RESULTADOS}

De los 167 pacientes con registro informático completo, a 9 se les realizaron 4 biopsias, a 30 pacientes 3 biopsias y a 128 únicamente 2 biopsias. Se excluyen 22 por habérseles practicado alguna de las biopsias previas en otro centro.

La muestra final de nuestra serie fue de 145 pacientes. Se trataba de pacientes con una edad media de 64 años, un PSA medio: 12,51 ng/ml, una media de PSA libre/total de 0,16 y de PSA densidad de 0,285; el volumen medio prostático de estos pacientes sobrepasaba los 50 cc. En los pacientes en los que finalmente se detectó cáncer, el tiempo medio entre la primera biopsia y la positiva fue de 15,8 meses, el número medio de cilindros por biopsia de próstata fue de 6,8; un 21,7 \% de los pacientes de nuestra serie tenía un tacto rectal sospechoso y en un $41 \%$ de los pacientes se objetivó un nódulo hipoecoico en la escala de grises. El patólogo responsable del examen de las muestras detectó en un 16,8\% de pacientes alguna lesión premaligna tipo PIN, en las biopsias negativas para tumor, así como un 20,3\% de displasia microacinar atípica (Tabla 1).
Tabla 1. Estudio descriptivo de las variables

\begin{tabular}{lcc}
\hline & Media & D.E. \\
\hline Edad (años) & 63,72 & 6,59 \\
PSA (ng/ml) & 11,80 & 26,69 \\
PSA cociente (PSAlibre/PSAtotal) & 0,16 & 0,09 \\
Volumen prostático (cm ${ }^{3}$ ) & 53,37 & 32,83 \\
Densidad PSA & 0,27 & 0,55 \\
N, Cilinindros & 6,55 & 1,47 \\
Velocidad del PSA ng/ml/año & 3,65 & 13,77 \\
Tiempo medio entre biopsias (días) & 620,77 & 546 \\
\hline & $\mathbf{n}$ & $\%$ \\
\hline Tacto rectal & 32 & 18,5 \\
Imagen sospechosa en la EG & 66 & 39,3 \\
Lesión AP premaligna & 57 & 30,2 \\
\hline
\end{tabular}

En esta serie, la tasa global de detección de cáncer para los pacientes a los que se les indicó una biopsia de repetición llegó al 46\%. A 39 pacientes se les indicó una tercera biopsia y a 9 de éstos les fue practicada una cuarta. La tasa de detección en las terceras biopsias fue del $25,7 \%$ y se detectó cáncer a 5 de los 9 pacientes con una cuarta.

En el análisis univariante, encontramos que las variables que influyen en la aparición de adenocarcinoma en biopsias de repetición son: Edad, densidad del PSA, tacto rectal, tamaño ecográfico, imágenes sospechosas según la escala de grises y lesiones premalignas en alguna de las biopsias previas (Tabla 2).

Cuando se realizó el análisis multivariante, las variables que demuestran capacidad predictiva, independiente, significativa, de riesgo de biopsia positiva son: edad, tacto rectal, tamaño ecográfico y presencia de lesión premaligna (Tabla 3).

La prueba de validación interna del estudio no es estadísticamente significativa $(p=0,389)$. Lo que permite aceptar el ajuste, ya que no hay discrepancias significativas entre frecuencias observadas y esperadas (Tabla 4). Para este estudio el índice de concordancia resultó ser 0.80 , lo que indica una fuerte habilidad discriminatoria por parte de éste.

Concretamente con el modelo hubiésemos clasificado correctamente el $71,9 \%$ de los pacientes de nuestra muestra (Tabla 5).

Mediante la resolución del mismo se ha llegado a crear un nomograma que está representado en la Figura 1. 
Tabla 2. Análisis estadístico univariante

\begin{tabular}{lccccccc}
\hline Variable & $\boldsymbol{\beta}$ & $\mathbf{E E}$ & Test Wald & valor de $\mathbf{p}$ & Odds.Ratio & O.R Inf 95\% & O.R Sup 95\% \\
\hline Edad & 0,108 & 0,026 & 16,902 & $\mathbf{0 , 0 0 1}$ & 1,114 & 1,058 & 1,173 \\
PSA & 0,020 & 0,023 & 0,761 & N.S & 0,370 & 0,008 & 17,335 \\
VelPSA & 0,020 & 0,024 & 0,706 & N.S & 1,02 & 0,973 & 1,070 \\
PSA libre/total & $-0,099$ & 1,963 & 0,257 & N.S & 0,370 & 0,008 & 17,335 \\
TR & 1,457 & 0,443 & 10,833 & $\mathbf{0 , 0 0 1}$ & 4,293 & 1,803 & 10,223 \\
Tamaño ECO & $-0,015$ & 0,006 & 6,459 & $\mathbf{0 , 0 1 1}$ & 0,986 & 0,975 & 0,997 \\
Dens. PSA & 3,197 & 1,166 & 7,522 & $\mathbf{0 , 0 0 6}$ & 24,459 & 2,49 & 240,272 \\
Tamaño AECO & $-0,007$ & 0,007 & 1,076 & N.S & 0,993 & 0,979 & 1,007 \\
Nod.hipo. & 0,744 & 0,321 & 5,361 & $\mathbf{0 , 0 2 1}$ & 2,104 & 1,121 & 3,948 \\
N.cilin. & $-0,196$ & 0,109 & 3,225 & N.S & 0,822 & 0,664 & 1,018 \\
Les.Premal. & 1,217 & 0,338 & 12,959 & $\mathbf{0 , 0 0 0}$ & 3,378 & 1,741 & 6,553 \\
\hline
\end{tabular}

N.S. No significativa

Tabla 3. Variables significativas según el modelo de regresión logística multivariante

\begin{tabular}{lccccccc}
\hline Variable & $\boldsymbol{\beta}$ & $\mathbf{e , s}$ & Wald & p.valor & Odds.Ratio & O.R Inf 95\% & O.R Sup 95\% \\
\hline Edad & 0,151 & 0,034 & 19,637 & 0,000 & 1,163 & 1,088 & 1,243 \\
TR & 1,305 & 0,555 & 5,526 & 0,019 & 3,686 & 1,242 & 10,939 \\
TamañoECO & $-0,017$ & 0,007 & 6,148 & 0,013 & 0,983 & 0,970 & 0,996 \\
Les.Premalig. & 0,978 & 0,401 & 5,957 & 0,015 & 2,659 & 1,212 & 5,830 \\
\hline
\end{tabular}

Tabla 4. Bondad de ajuste del modelo. Prueba de Hosmer y Lemeshow

\begin{tabular}{lcc}
\hline Chi-cuadrado & Grados de libertad & Valor de $\mathbf{p}$ \\
\hline 8,583 & 8 &, 379 \\
\hline
\end{tabular}

Tabla 5. Validación interna del modelo. Tabla de clasificación

\begin{tabular}{lccc}
\hline Observado & \multicolumn{3}{c}{ Pronosticado } \\
& $\begin{array}{c}\text { Biopsia } \\
\text { negativa }\end{array}$ & $\begin{array}{c}\text { Biopsia } \\
\text { positiva }\end{array}$ & $\begin{array}{c}\text { Porcentaje } \\
\text { correcto }\end{array}$ \\
\hline Biopsia negativa & 62 & 21 & 74,7 \\
Biopsia positiva & 24 & 53 & 68,8 \\
Porcentaje global & & & 71,9 \\
\hline
\end{tabular}

\section{DISCUSION}

Un problema cada vez más frecuente en nuestra práctica diaria es la imposibilidad de afirmar de manera absoluta que una biopsia negativa para cáncer sea un verdadero negativo. Según las guías clínicas europeas es necesario un seguimiento del paciente con las variables pronosticas disponibles, y la decisión de repetir la biopsia será personalizada y consensuada entre clínico y paciente ${ }^{7}$. Todo esto suele provocar una situación de estrés para el paciente que debe estar al corriente de que quizás su caso sea un falso negativo o de que independientemente de que no tuviera cáncer en el momento de la biopsia pudiera desarrollarlo en un futuro.

Por otro lado las series más extensas refieren que el 91\% de los cánceres de próstata son diagnosticados en la primera y segunda biopsia, y en las sucesivas la tasa de detección desciende de manera importante ${ }^{8}$. Para evitar esta reducción en el rendimiento de la biopsia, muchos autores proponen cambiar las estrategias de biopsia de próstata. Accesos transperineales en lugar del clásico transrectal $^{9}$; o bien, aumentar el número de cilindros hasta realizar lo que Stewart et al. ${ }^{10}$ denominaron la biopsia de saturación obteniendo hasta 45 cilindros bajo anestesia general y con un mapeo radial desde el cuerno lateral hacia la zona más medial; las biopsias que incluyen la zona transicional, donde Lui et al. ${ }^{11}$. encontraron hasta un 53\% de tumores para pacientes con biopsias previas negativas; estrategias mixtas que combinan el acceso transperineal 


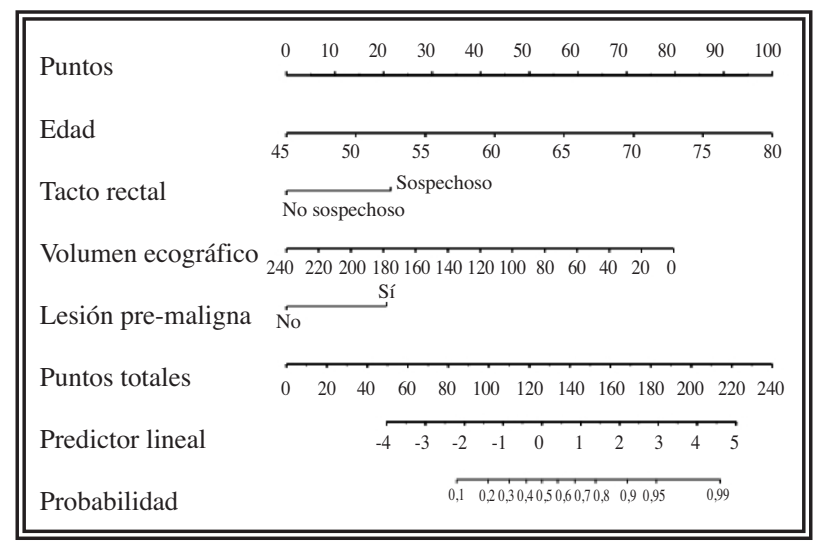

FIGURA 1. Nomograma para pacientes con sospecha de cáncer de próstata y biopsia previa negativa para cáncer. Variables: Edad; Tacto rectal; Volumen ecográfico prostático medido transrectalmente; Lesión premaligna (lesión histológica tipo PIN o proliferación microacinar atípica. Cada variable tiene un valor en la escala que le acompa$\tilde{n} a$, dicho valor se corresponde con una puntuación (Puntos). El sumatorio de las puntuaciones de las cuatro variables (Puntos Totales) traduce una probabilidad.

con el transrectal ${ }^{12}$; y finalmente otros que proponen la resección transuretral con la biopsia transrectal de las zonas lateroprostáticas ${ }^{13}$. Todos estos trabajos son, a nuestro juicio, necesarios pero criticables puesto que podrían incurrir en el sobrediagnóstico y consecuente sobretratamiento del cáncer de próstata y al aumento de la morbilidad de esta prueba diagnóstica.

Independientemente de los intentos en la mejora de la estrategia de biopsia, otros grupos han dirigido sus esfuerzos a seleccionar de forma correcta a los pacientes por grupos de mayor o menor riesgo.

En este sentido son varios los grupos que han estudiado el rendimiento de las variables que se utilizan para el despistaje del cáncer de próstata, en este subgrupo de pacientes con biopsias previas negativas.

Así Fowler et al. ${ }^{14}$ sobre una serie de pacientes con biopsias de repetición objetivaron que los factores con mayor capacidad predictiva eran el cociente PSA total/PSA libre y la edad, mientras que no encuentran relevancia de variables como el PSA densidad, velocidad del PSA o lesiones premalignas en los análisis histológicos de las biopsias previas. Otro grupo, el de Keetch et al. ${ }^{15}$ observan que los principales factores predictores son la velocidad del PSA y el PSA densidad. En su trabajo, en el grupo de pacientes con una densidad de PSA mayor de 0,15 y un aumento de los valores del PSA mayor de $0,75 \mathrm{ng} / \mathrm{dl}$ anual, la rentabilidad de la biopsia de repetición era del $46 \%$.
El grupo de Yanke et al. ${ }^{16}$, ha desarrollado y validado un nomograma para seleccionar aquellos pacientes con alto riesgo de cáncer de próstata. Presentaron un complejo modelo que incluye 10 variables: Edad, tacto rectal, número de cililindros de las biopsias previas, antecedentes de lesiones PIN y de proliferación microacinar atípica, PSA, velocidad de PSA, antecedentes familiares de cáncer prostático, tiempo en meses desde la primera biopsia y tiempo en meses desde la biopsia anterior. Por el contrario su modelo no incluía el PSAlibre/PSA total, ni el volumen de la próstata. Con una tasa de ajuste del $70,8 \%$, mejoraba la capacidad predictiva del PSA total en un $4 \%$ o de la velocidad del PSA en un $10 \%$. La complejidad del modelo y la escasa mejora en la capacidad predictiva, respecto a cada una de las variables clásicas utilizadas individualmente, hacen que por el momento no tenga una aplicación clínica generalizada. La muestra sobre la que desarrollan el modelo incluye un 55\% de pacientes de raza negra, y pese a que este mismo grupo en otro trabajo trató de demostrar que este hecho no tenía relevancia en el modelo ${ }^{17}$, es probablemente un inconveniente para la aplicación en nuestro medio.

Al trabajar con el controvertido grupo de pacientes con biopsia previa negativa, los resultados son, en ocasiones, diferentes a lo esperado y en nuestra serie, el PSA total, el PSA libre/total y la velocidad del PSA no influyen en la aparición de cáncer, tanto en el estudio univariante como en el multivariante. Tampoco el número de cilindros es estadísticamente significativo; probablemente porque la mayor parte de las biopsias incluidas en esta serie se realizaban mediante toma de seis cilindros, incluso para las segundas biopsias; de hecho, la media de cilindros por biopsia era de 6,55 $\pm 1,54$. Sólo los últimos pacientes incluidos en este estudio se biopsiaron con el protocolo vigente actualmente en nuestro servicio, (10 cilindros para la primera biopsia, 14 cilindros para segunda biopsia y 18 para tercera y sucesivas) ${ }^{18}$. Además durante ese periodo de tiempo la sensibilidad de las primeras biopsias, probablemente, no fue lo alta que debiera, y de ahí la alta tasa diagnóstica (un 46\%) de las biopsias de repetición en nuestra serie.

Cuando aplicamos el análisis multivariante, dejan de ser estadísticamente significativas variables como PSA densidad y las lesiones hipoecoicas o sospechosas según la escala de grises en la ecografía transrectal. 
Es contradictorio el hecho de que el volumen prostático influya en la aparición de cáncer y no lo haga la densidad del PSA; probablemente, conforme el trabajo tenga mayor potencia estadística, el modelo ajuste más y la densidad del PSA resulte significativa.

En los pacientes a los que se somete a una primera biopsia el valor de los hallazgos ecográficos es controvertido, y aunque las áreas hipoecoicas en los ultrasonidos tienen 2 veces más probabilidad de corresponder a un cáncer que las izo ecoicas, la mayoría de las lesiones hipoecoicas no son cánceres, y el 50\% de los cánceres no palpables con un diámetro menor de $1 \mathrm{~cm}$ no se observan en la ecografía $^{19}$. Por tanto no es extraño que el modelo no incluya esta variable.

Las variables a partir de las cuales desarrollamos el nomograma son: edad, tacto rectal, la aparición de una lesión pre-neoplásica tipo PIN o proliferación microacinar atípica y el volumen prostático.

La incidencia de cáncer de próstata aumenta con la edad y esta máxima no es una excepción en este grupo de pacientes, no obstante esto no influirá en el sentido común del clínico que deberá obviar biopsias en pacientes que no se beneficiarían del diagnóstico.

El tacto rectal es prioritario en el despistaje del cáncer de próstata. Así lo refieren todas las guías clínicas, aunque actualmente se discute el valor del mismo en pacientes jóvenes con valores bajos del PSA, en nuestro trabajo es la variable con mayor poder predictivo ${ }^{3}$ (Tabla 3 ).

Son muchos los trabajos que han descrito la relación de las lesiones PIN y proliferación microacinar atípica con la presencia de cáncer. La OMS reconoce la lesión tipo PIN como la lesión pre-invasiva del cáncer de próstata ${ }^{2}$ Y su presencia se asocia al cáncer de próstata en el 27-50\% de los casos según las series ${ }^{20}$. La proliferación microacinar atípica se asocia a la presencia de cáncer en biopsias sucesivas en alrededor del $50 \%$ de los pacientes ${ }^{2}$. Por todo ello era de esperar que, en nuestra serie, la variable "lesiones premalignas" que engloba a ambas, tuviera valor predictivo. Por último, también en los pacientes con biopsias previas negativas, la edad se relaciona con el aumento de la incidencia del cáncer de próstata, y aunque esta variable esté presente en el modelo, es evidente que a personas con una corta esperanza de vida no se les debe proponer la biopsia de próstata.
Según nuestros resultados el volumen prostático es un factor protector, a mayor volumen menos probabilidad de detectar cáncer OR=0,983. En la literatura está descrita la influencia del volumen prostático en la detección del cáncer aunque no queda claro si es un motivo de probabilidad (tejido biopsiado en gramos/tejido prostático total) o si el adenoma de la zona transicional influye en la fisiopatología de la aparición del cáncer ${ }^{21}$

El hecho de que en 5 de 9 pacientes a los que se indica una cuarta biopsia el resultado sea positivo, lo consideramos anecdótico, pues probablemente este subgrupo esté sesgado ya que sólo se indican estas biopsias a pacientes con una firme sospecha de cáncer.

Este trabajo, pese a tener una correcta validación interna, al realizarse sobre una muestra histórica de pacientes, es retrospectivo. Estos modelos estadísticos son dinámicos, es decir, se ajustarán y perfeccionarán cuando ampliemos de forma prospectiva la serie, sobre la que se desarrolla el modelo. Además será necesaria una validación de los resultados con un grupo de pacientes externos a la serie con la que se diseñó el modelo.

\section{CONCLUSIONES}

Mientras la biopsia prostática sea la prueba de elección para el diagnóstico de cáncer prostático, biopsiar por segunda o tercera vez a un paciente es una decisión difícil que no debería de ser tomada en función de una sola variable. Todos los trabajos comentados tratan de ayudar en dicha detección. Hasta la aparición de nuevas variables, probablemente marcadores moleculares, con un alto valor pronóstico, lo ideal sería contar con un nomograma de alta capacidad discriminativa que facilite la decisión de indicar una biopsia de repetición.

\section{REFERENCIAS}

1. Applewhite JC, Matlaga BR, McCullough DL. Results of the 5 region prostate biopsy method: the repeat biopsy population. J Urol. 2002;168(2):500-503.

2. Bostwick DG, Qian J, Schlesinger C. Contemporary pathology of prostate cancer. Urol Clin North Am. 2003;30(2):181-207.

3. Moul JW. Population screening for prostate cancer and emerging concepts for young men. Clin Prostate Cancer. 2003;2(2):8797.

4. Hara I, Miyake H, Hara S, Arakawa S, Kamidono S. Differential involvement of the Fas receptor/ligand system in p53-dependent apoptosis in human prostate cancer cells. Prostate. 2000;45(4): 341-349. 
5. Garzotto M, Hudson RG, Peters L, Hsieh YC, Barrera E, Mori $\mathrm{M}$, et al. Predictive modeling for the presence of prostate carcinoma using clinical, laboratory, and ultrasound parameters in patients with prostate specific antigen levels $<$ or $=10 \mathrm{ng} / \mathrm{mL}$. Cancer. 2003;98(7): 1417-1422.

6. Suzuki H, Komiya A, Kamiya N, Imamoto T, Kawamura K, Miura J, et al. Development of a nomogram to predict probability of positive initial prostate biopsy among Japanese patients. Urology. 2006;67(1):131-136.

7. Aus G, Abbou CC, Bolla M, Heidenreich A, Schmid HP, van Poppel $\mathrm{H}$, et al. EAU guidelines on prostate cancer. Eur Urol. 2005;48(4):546-551.

8. Roehl KA, Antenor JA, Catalona WJ. Serial biopsy results in prostate cancer screening study. J Urol. 2002;167(6):24352439.

9. Furuno T, Demura T, Kaneta T, Gotoda H, Muraoka S, Sato T, et al. Difference of cancer core distribution between first and repeat biopsy: In patients diagnosed by extensive transperineal ultrasound guided template prostate biopsy. Prostate. 2004;58(1):76-81.

10. Stewart CS, Leibovich BC, Weaver AL, Lieber MM. Prostate cancer diagnosis using a saturation needle biopsy technique after previous negative sextant biopsies. J Urol. 2001;166(1):86-91; discussion 91-92.

11. Lui PD, Terris MK, McNeal JE, Stamey TA. Indications for ultrasound guided transition zone biopsies in the detection of prostate cancer. J Urol. 1995;153(3 Pt 2):1000-1003.

12. Kawakami S, Okuno T, Yonese J, Igari T, Arai G, Fujii Y, et al. Optimal sampling sites for repeat prostate biopsy: a recursive partitioning analysis of three-dimensional 26-core systematic biopsy. Eur Urol 2007;51(3):675-682; discussion 682-683.

13. Puppo P, Introini C, Calvi P, Naselli A. Role of transurethral resection of the prostate and biopsy of the peripheral zone in the same session after repeated negative biopsies in the diagnosis of prostate cancer. Eur Urol. 2006;49(5):873-878.

14. Fowler JE, Jr., Bigler SA, Miles D, Yalkut DA. Predictors of first repeat biopsy cancer detection with suspected local stage prostate cancer. J Urol. 2000;163(3):813-818.
15. Keetch DW, Humphrey P, Stahl D, Smith DS, Catalona WJ. Morphometric analysis and clinical followup of isolated prostatic intraepithelial neoplasia in needle biopsy of the prostate. $J$ Urol. 1995; 154(2 Pt 1):347-351.

16. Yanke BV, Gonen M, Scardino PT, Kattan MW. Validation of a nomogram for predicting positive repeat biopsy for prostate cancer. J Urol. 2005;173(2):421-424.

17. Yanke BV, Salzhauer EW, Colon I. Is race a positive predictor of cancer on repeat prostate biopsy?. J Urol. 2006;176(3):11141117.

18. Ramirez Backhaus M, Trassierra Villa M, Arlandis Guzman S, Bosquet Sanz M, Pontones Moreno JL, Jimenez Cruz JF. Yield diagnosis of the peripheral cores in 10 needle extended prostate biopsy. Actas Urol Esp. 2007;31(1):11-16.

19. Ellis WJ, Chetner MP, Preston SD, Brawer MK. Diagnosis of prostatic carcinoma: the yield of serum prostate specific antigen, digital rectal examination and transrectal ultrasonography. J Urol. 1994;152(5 Pt 1):1520-1525.

20. Rodriguez-Patron Rodriguez R, Mayayo Dehesa T, Burgos Revilla FJ, Sanz Mayayo E, Garcia Gonzalez R. Prognostic significance of PIN and Atypical Small Acinar Proliferation on transrectal ultrasound-guided prostate biopsy. Actas Urol Esp. 2006;30(4):359-366.

21. Bruno JJ, 2nd, Armenakas NA, Fracchia JA. Influence of prostate volume and percent free prostate specific antigen on prostate cancer detection in men with a total prostate specific antigen of 2.6 to $10.0 \mathrm{ng} / \mathrm{ml}$. J Urol. 2007;177(5):1741-1744.

Correspondencia autor: Dr. M. Ramírez Backhaus Servicio de Urología

Hospital Universitario La Fe

Avda. Campanar $\mathrm{n}^{\circ} 21,46009$. Valencia

Tel.: 963862700

e-mail: ramirezbackhaus@yahoo.es

Información artículo: Original - Cáncer de próstata

Trabajo recibido: julio 2007

Trabajo aceptado: noviembre 2007 